Aus dem Universitätsinstitut für Untersuchungen mit Röntgenstrahlen in Berlin.

\section{Ueber einen neuen Kinematographen zur Diagnostik mittels Röntgenstrahlen bei inneren Leiden.}

\section{Von E. Grunmach.}

Auf der diesjährigen Naturforscher-Versammlung in Karlsruhe wurden die Ergebnisse der kinematographischen Röntgenuntersuchung innerer Organe, insbesondere des Magens und Darms, von mir geschildert. Bei dieser Untersuchung kam das an Gesunden und Kranken im Universitätsinstitut von mir erprobte Cer-Thorpräparat ${ }^{1}$ ) zur Anwendung, da durch dieses schon bei einem Drittel der Dosis von Bismutum carbonicum und Zinkonoxyd dieselben Leistungen für die Diagnostik, aber außerdem noch therapeutische Erfolge bei Gastralgien erzielt werden konnten.

Die Ausführung der zahlreichen Röntgenversuche fand in der Weise statt, daß zunächst nach der Magenfüllung mit Griesbrei und dem Cer-Thorpräparat halbstündlich auf dem Leuchtschirm die Lage, Form und Bewegung des Magens, darauf nach drei bis vier Stunden die Gestalt und Inhaltsbewegung des Darms beobachtet sowie dessen Verhalten sechs bis sieben Stunden nach der Nahrungsaufnahme bei Atemstillstand kinematographisch mittels X-Strahlen an Gesunden und Kranken festgestellt wurde. - Zu diesen diagnostischen-Versuchen bewährte sich mein neuer, durch den Ingenieur Klemp hergestellter Röntgen-Kinematograph, dessen Konstruktion und Leistung aus der Beschreibung der drei Figuren A, B und $\mathrm{C}$ zu ersehen sein dürfte.-Bei diesem Appa-

Fig. A.

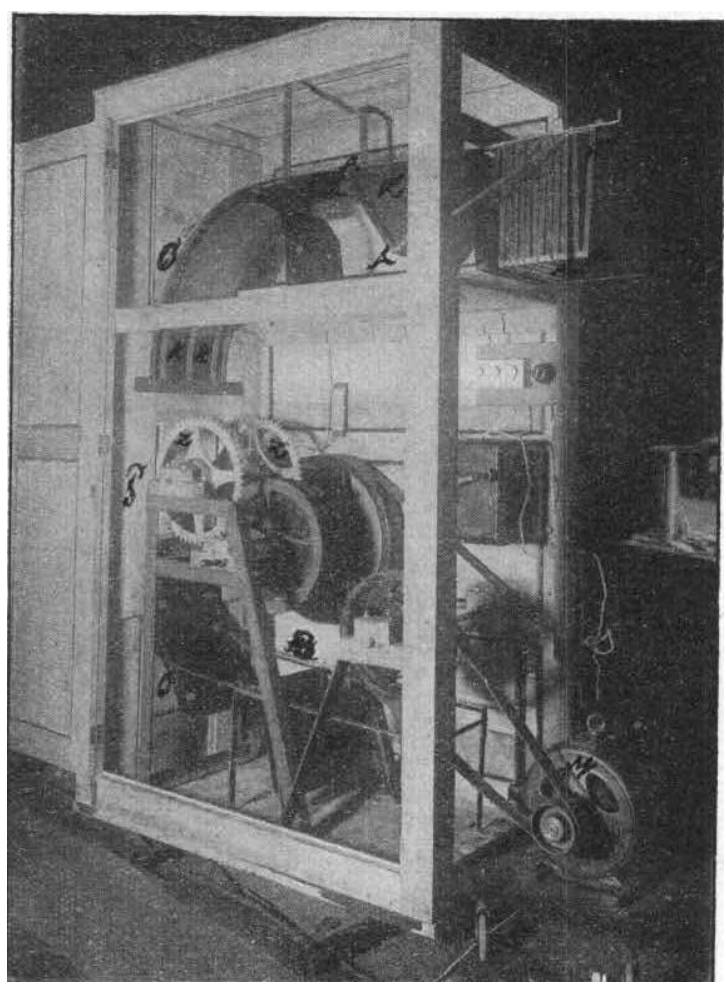

rate werden nämlich durch einen Elektromotor die plattentragenden Kassetten (K), die durch Gelenke (A) fächerartig untereinander verbunden und seitlich mit einer Verzahnung $(O)$ versehen sind, an Führungsschienen (B) entlang von oben herabgeleitet, sowie durch Eingreifen von Zahnrädern $(Z)$ in die Verzahnung der Kassetten zur Aufnahmefläche (P) der Versuchsperson gefiihrt, wo sie nach elektrischer Oeffnung des Momentverschlusses (V) den X-Strahlen exponiert, alsbald wieder fächerartig untereinander verbunden, an Führungsschienen (B) herabgleiten. Insbesondere sei noch bemerkt,

1) Internationale Beiträge zur Pathologie und Therapie der Ernährungsstörungen. Bd. 3, H. 2 daß zum elektrischen Kassetten- und Plattenwechsel die erprobte Maltheserkreuzkonstruktion verwertet wird. Die

Fig. B 1.. C.
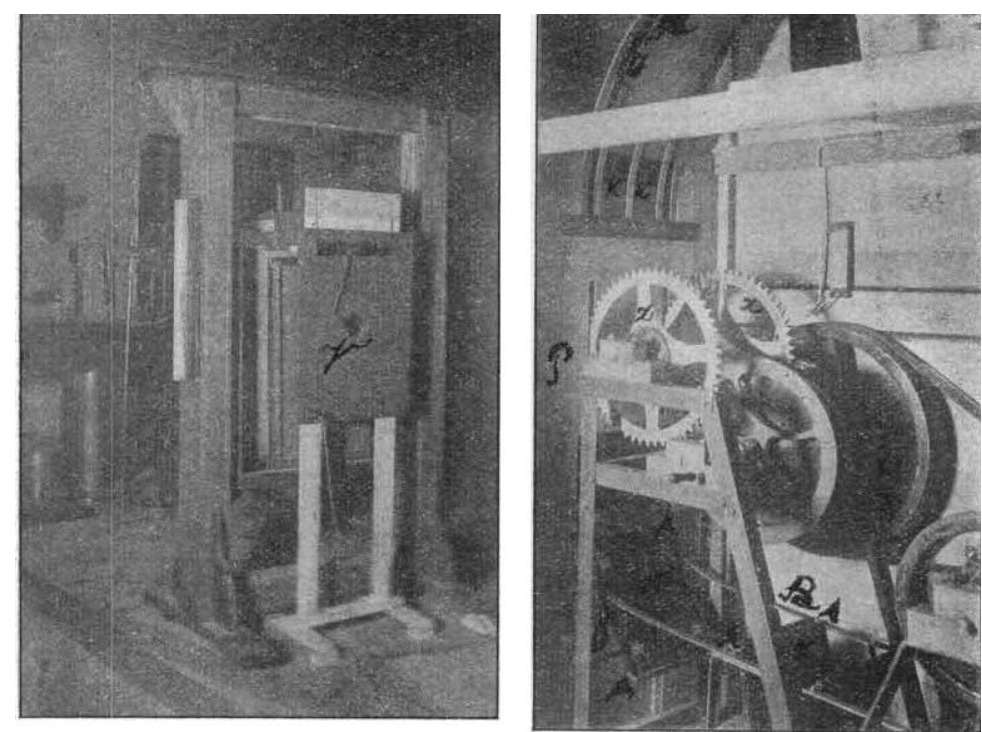

Vorzüge des neuen Apparates bestehen im wesentlichen darin: daß 1. Röntgenplatten statt Films angewandt und dadurch die einzelnen Aufnahmen $u m$ zwei Drittel billiger als bisher werden, 2. Platten in der Größe von [24:30 cm und auch kleinere zu benutzen sind, 3. große Veränderung der Geschwindigkeit beim Transport der einzelnen Kassetten durch Einstellung des Regulierwiderstandes möglich ist, sodaß 1-10 Aufnahmen in einer Sekunde sich machen lassen, 4. eine unbegrenzte Zahl der hintereinander folgenden Aufnahmen stattfinden kann.

So gestattet der im Universitätsinstitut angewandte Apparat 36 Aufnahmen mittels der X-Strahlen kinematographisch auszuführen.

Zum Betriebe des neuen Apparats ${ }^{1}$ ) wurden außerdem die neuen Radiologie-Starkstromröhren und als Verstärkungsschirme die bekannten Radiologiefolien neben dem SiemensInduktor von einem Meter Funkenlänge und dem elektrolytischen Unterbrecher in Anwendung gebracht.

Der beschriebene Röntgen-Kinomatograph dient aber nicht allein zur Aufnahme der Bewegungen des Magens und Darms sowie der übrigen inneren Organe, z. B. des Zwerchfells, Herzens, der Brustaorta und Speiseröhre, sondern kann auch für die Chirurgie, z. B. zum Studiun der Gelenke an den Extremitäten unter normalen und pathologischen Bedingungen, lehrreiche Anwendung finden.

Zum Beweise für die Leistungsfähigkeit des neuen Apparats zur Diagnostik in nerer Leiden seien hier unter den kinematographisch mittels X-Strahlen aufgenommenen Bildern des Magen-Darmkanals nur von zwei fast gleichaltrigen Individuen, einem sonst gesunden jungen Mann von 27 Jahren mit gesenktem Colon transversum und einem jungen Mädchen von 26 Jahren mit Gastroenteroptose, die sechs bis sieben Stunden nach der Nahrungsaufali me bei Atemstillstand gewonnenen Kinematogramme des Darms demonstriert.

Bei der ersten Versuchsperson (18 Darmaufnahmen, jede bei ein Sechstelsekunde Exposition) sah man schon im Bereich des gesenkten Colon transversum den Inhalt desselben bald als zusammenhängende Masse, bald in Knollen zerstückelt vorund rückwärts hin- und hergeworfen werden, den Darmumfang nach der Flexura hepatica hin sich erweitern und von hier zur Flexura lienalis schlangenförmig sich verschmälern, kurz Bewegungen in der längs- und Querrichtung des Dickdarms mit der Hauptkraft, den Inhalt immer weiter vorwärts ins Colon descendens zu befördern.

Dagegen fand man bei den von der zweiten Versuchsperson sechs bis sieben Stunden nach der Nahrungsaufnahme

1) Der neue Apparat wird rom Medizinischen Warenhaus (ActienGesellschaft) Berlin geliefert. 
gewonnenen Kinematogrammen (18 Darmaufnahmen, jede bei ein Sechstelsekunde Exposition) den Darminhalt noch im tief gesenkten Ileum liegen, das zu einem Knäuel aufgerollt sich nur wenig in der Mitte, aber stärker in den Anfangspartien
Zur Dlustration der geschilderten Resultate sollen an dieser Stelle von jeder Versuchsperson nur vier hintereinander gewonnene Aktinogramme in $1 / 5$ Größe zur Darstellung gelangen, von denen die Abbildungen 5-8 dem
Fig. 5.

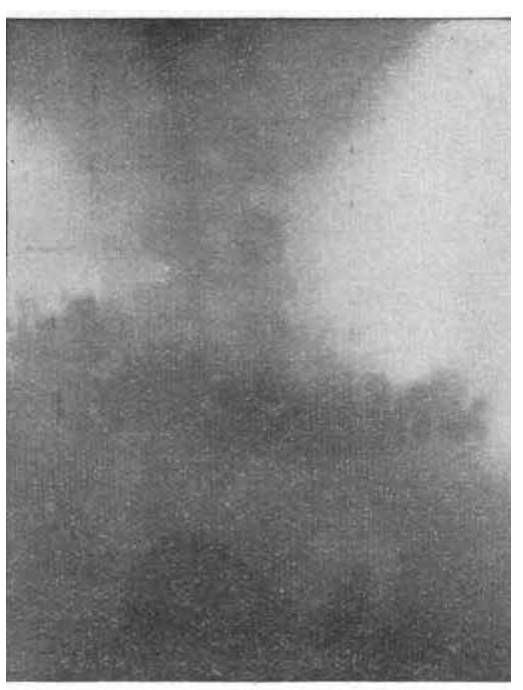

Fig. 8.

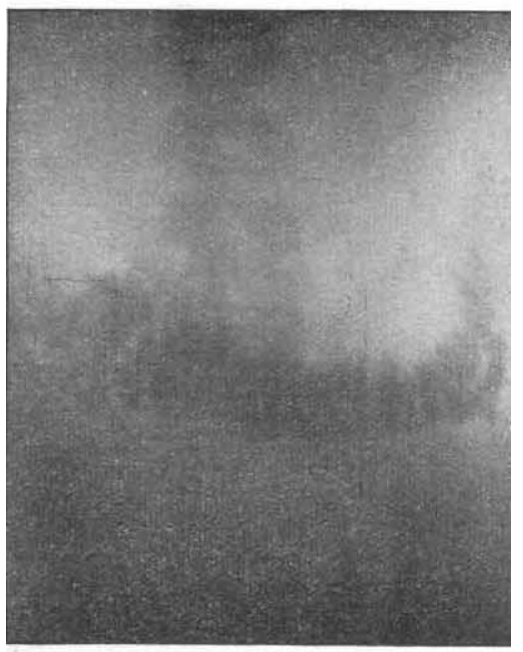

Fig. III.

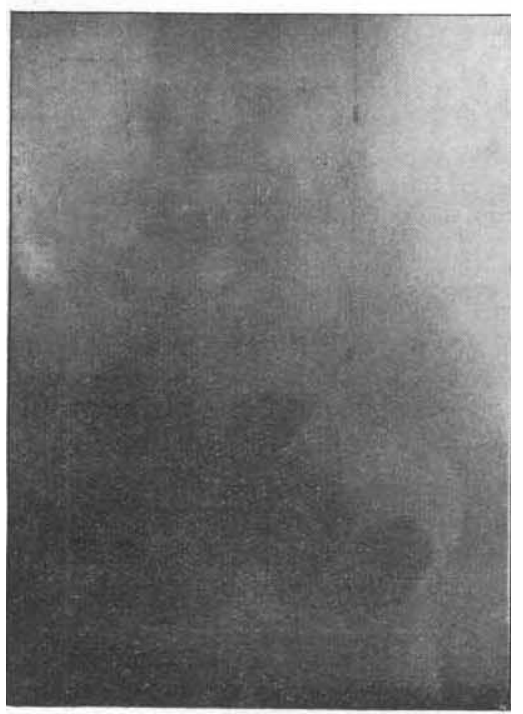

Fig. 6.

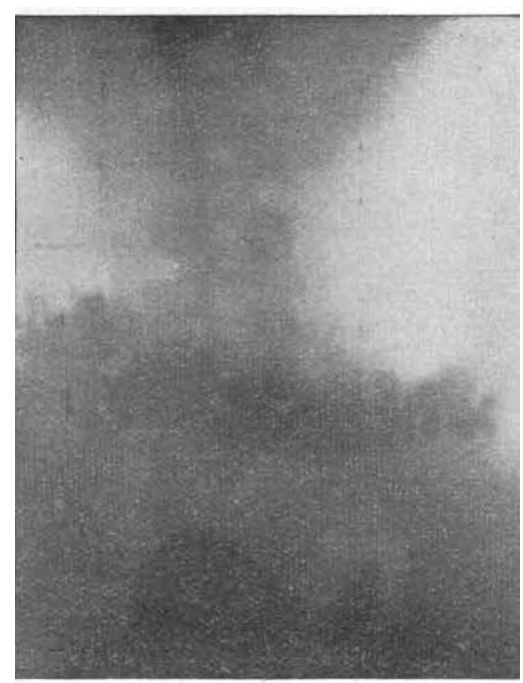

Fig. 8.

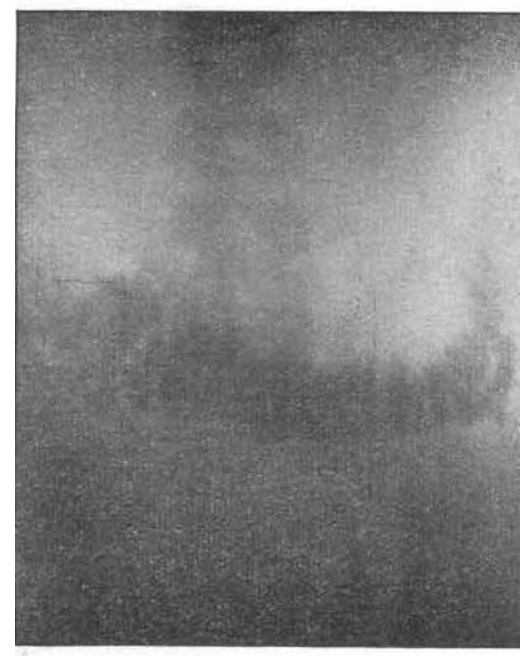

Fig. III.

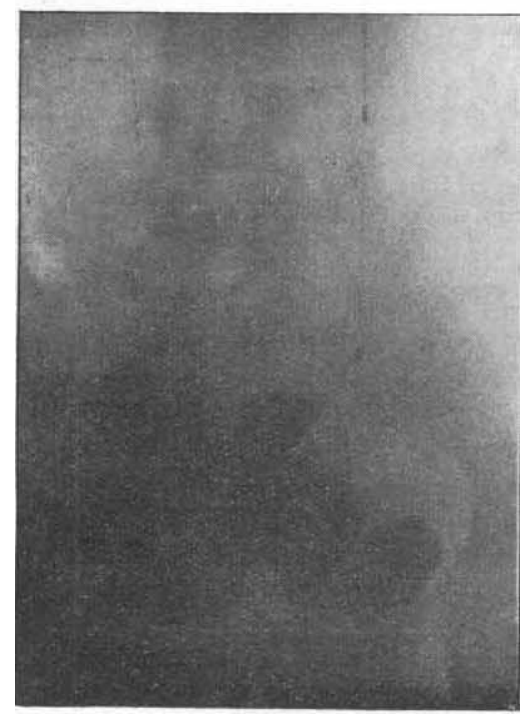

Fig. 7.

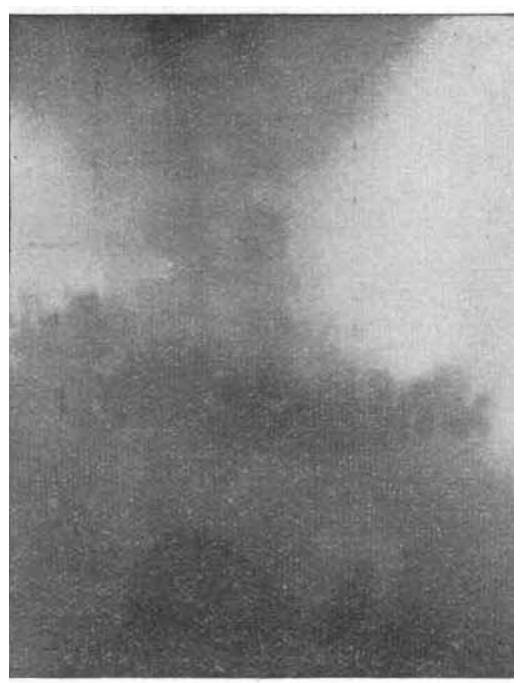

Fig. 8.

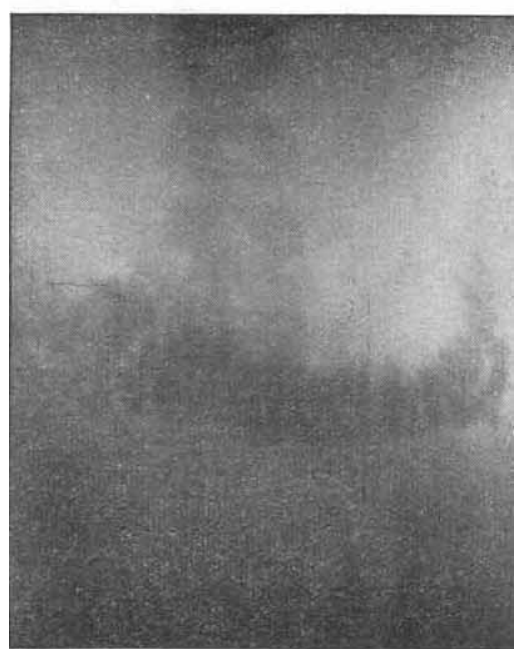

Fig. III.

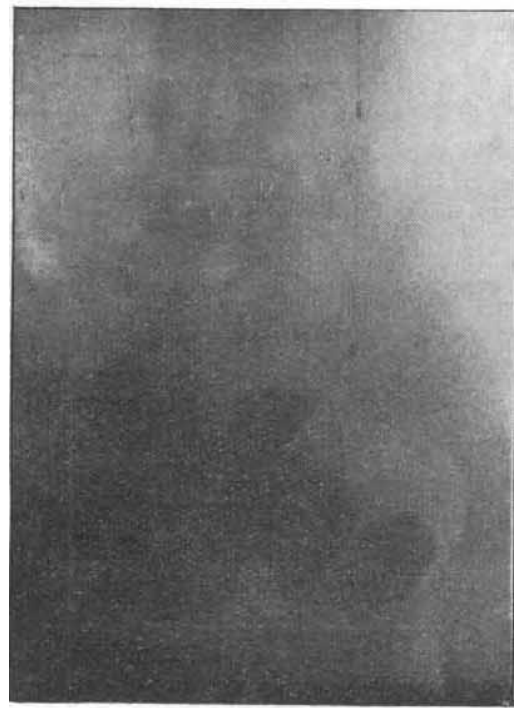

bewegte und aus dem während der ganzen kinematographischen Untersuchung der Darminhalt nur wenige Zentimeter hoch ins Colon ascendens emporstieg, nachdem der Inhalt wiederholt Bewegungen nach vorwärts und rückwärts gemacht hatte. jungen Mann, I--TV dem jungen Mädchen entnommen sind. - Von der Patientin werden jedoch auch noch zur Vervollständigung des Gesamtbildes der Erkrankung die ersten vier Aktinogramme a. b. c. d. der kinematographischen Unter- 
Fig. b.

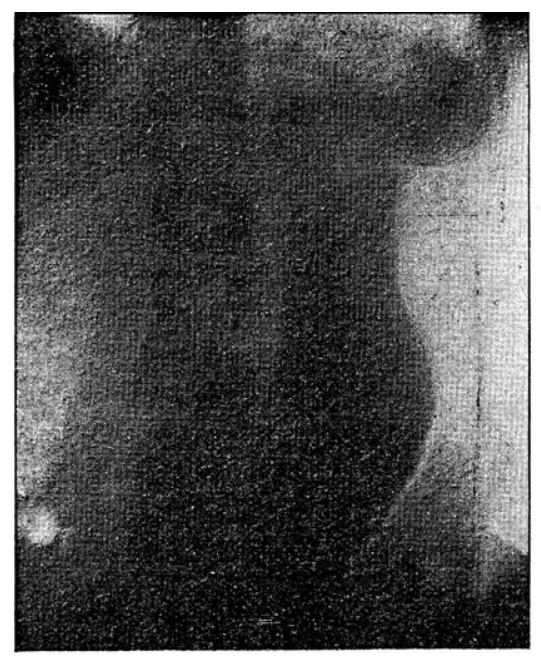

Fig. c.

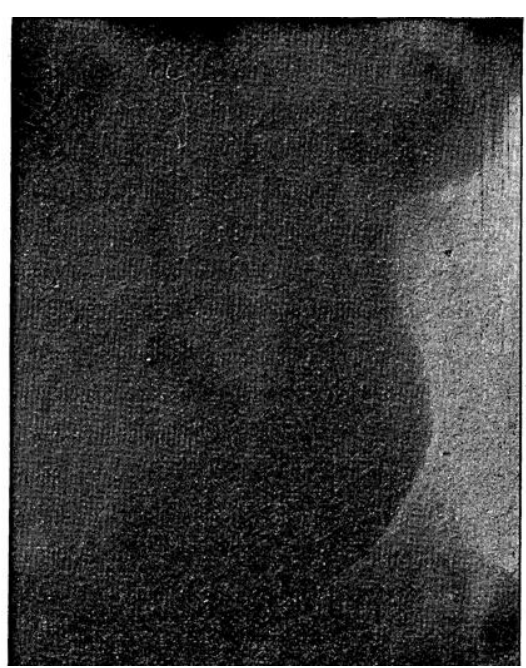

Fig. d.

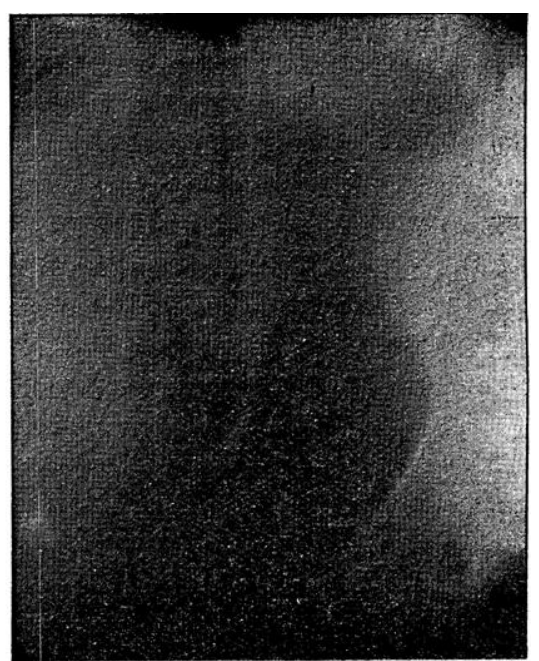

suchung des Magens bald nach der Kontrastmahlzeit (von den 18 Aufnahmen, jede bei ein Sechstelsekunde Exposition) hier wiedergegeben. Aus diesen Aktinogrammen lassen sich schon zunächst deutlich die Gastroptose, ferner die Form und Bewegungen des ganzen Magens, insbesondere die Gestaltsveränderungen der Pars pylorica, daneben aber auch die Lage und ausgeprägte Form der Pars horizontalis superior sowie der descendens duodeni ersehen.

Auf Grund der mit dem neuen Apparat erzielten Resuitate erwies sich also die Kinematographie mittels Röntgenstrahlen als eine wichtige Untersuchungsmethode zur Diagnostik innerer Krankheiten. 